\title{
Quantum Excitations in Quantum Spin Ice
}

\author{
Kate A. Ross, ${ }^{1}$ Lucile Savary, ${ }^{2}$ Bruce D. Gaulin, ${ }^{1,3,4}$ and Leon Balents ${ }^{5, *}$ \\ ${ }^{1}$ Department of Physics and Astronomy, McMaster University, Hamilton, Ontario, L8S 4M1, Canada \\ ${ }^{2}$ Ecole Normale Supérieure de Lyon, 46, allée d'Italie, 69364 Lyon Cedex 07, France \\ ${ }^{3}$ Canadian Institute for Advanced Research, 180 Dundas St. W., Toronto, Ontario, M5G 1Z8, Canada \\ ${ }^{4}$ Brockhouse Institute for Materials Research, McMaster University, Hamilton, Ontario, L8S 4M1, Canada \\ ${ }^{5}$ Kavli Institute for Theoretical Physics, University of California, Santa Barbara, California, 93106-4030, USA
}

(Received 22 July 2011; published 3 October 2011)

\begin{abstract}
Recent work has highlighted remarkable effects of classical thermal fluctuations in the dipolar spin ice compounds, such as "artificial magnetostatics," manifesting as Coulombic power-law spin correlations and particles behaving as diffusive "magnetic monopoles." In this paper, we address quantum spin ice, giving a unifying framework for the study of magnetism of a large class of magnetic compounds with the pyrochlore structure, and, in particular, discuss $\mathrm{Yb}_{2} \mathrm{Ti}_{2} \mathrm{O}_{7}$, and extract its full set of Hamiltonian parameters from high-field inelastic neutron scattering experiments. We show that fluctuations in $\mathrm{Yb}_{2} \mathrm{Ti}_{2} \mathrm{O}_{7}$ are strong, and that the Hamiltonian may support a Coulombic "quantum spin liquid" ground state in low magnetic fields and host an unusual quantum critical point at larger fields. This appears consistent with puzzling features seen in prior experiments on $\mathrm{Yb}_{2} \mathrm{Ti}_{2} \mathrm{O}_{7}$. Thus, $\mathrm{Yb}_{2} \mathrm{Ti}_{2} \mathrm{O}_{7}$ is the first quantum spin liquid candidate for which the Hamiltonian is quantitatively known.
\end{abstract}

DOI: 10.1103/PhysRevX.1.021002

Rare-earth pyrochlores display a diverse set of fascinating physical phenomena [1]. One of the most interesting aspects of these materials from the point of view of fundamental physics is the strong frustration experienced by coupled magnetic moments on this lattice. The best explored materials exhibiting this frustration are the "spin ice" compounds, $\mathrm{Ho}_{2} \mathrm{Ti}_{2} \mathrm{O}_{7}, \mathrm{Dy}_{2} \mathrm{Ti}_{2} \mathrm{O}_{7}$, in which the moments can be regarded as classical spins with a strong easyaxis (Ising) anisotropy [2,3]. The frustration of these moments results in a remarkable classical spin liquid regime displaying Coulombic correlations and emergent "magnetic monopole" excitations that have now been studied extensively in theory and experiment [4-6].

Strong quantum effects are absent in the spin ice compounds, but can be significant in other rare-earth pyrochlores. In particular, in many materials the low-energy spin dynamics may be reduced to that of an effective spin $S=1 / 2$ moment, with the strongest possible quantum effects expected. In this case symmetry considerations reduce the exchange constant phase space of the nearestneighbor exchange Hamiltonian to a maximum of three dimensionless parameters [7]. The compounds $\mathrm{Yb}_{2} \mathrm{Ti}_{2} \mathrm{O}_{7}$, $\mathrm{Er}_{2} \mathrm{Ti}_{2} \mathrm{O}_{7}, \mathrm{Pr}_{2} \mathrm{Sn}_{2} \mathrm{O}_{7}[1]$ (and possibly $\mathrm{Tb}_{2} \mathrm{Ti}_{2} \mathrm{O}_{7}$ [8]) are of this type, and it has recently been argued that the spins in $\mathrm{Yb}_{2} \mathrm{Ti}_{2} \mathrm{O}_{7}$ and $\mathrm{Er}_{2} \mathrm{Ti}_{2} \mathrm{O}_{7}$ are controlled by exchange coupling rather than by the long-range dipolar interactions

\footnotetext{
*Corresponding author balents@kitp.ucsb.edu

Published by the American Physical Society under the terms of the Creative Commons Attribution 3.0 License. Further distribution of this work must maintain attribution to the author(s) and the published article's title, journal citation, and DOI.
}

Subject Areas: Magnetism, Strongly Correlated Materials

which dominate in spin ice $[9,10]$. This makes these materials beautiful examples of highly frustrated and strongly quantum magnets on the pyrochlore lattice. They are also nearly ideal subjects for detailed experimental investigation, existing as they do in large high-purity single crystals, and with large magnetic moments amenable to neutron scattering studies. $\mathrm{Yb}_{2} \mathrm{Ti}_{2} \mathrm{O}_{7}$ is particularly appealing because its lowest Kramers doublet is extremely well separated from the first excited one [11], and a very large single-crystal neutron scattering data set is available, allowing us to determine the full Hamiltonian quantitatively, as we will show. Although we specialize to $\mathrm{Yb}_{2} \mathrm{Ti}_{2} \mathrm{O}_{7}$ in the present article, the theoretical considerations and parameter determination method described here will very generally apply to all pyrochlore materials where exchange interactions dominate, and whose dynamics can be described by that of a single doublet.

Theoretical studies have pointed to the likelihood of unusual ground states of quantum antiferromagnets on the pyrochlore lattice $[12,13]$. Most exciting is the possibility of a quantum spin liquid (QSL) state, which avoids magnetic ordering and freezing even at absolute zero temperature, and whose elementary excitations carry fractional quantum numbers and are decidedly different from spin waves [14]. Although one neutron study [15] supported ferromagnetic order in $\mathrm{Yb}_{2} \mathrm{Ti}_{2} \mathrm{O}_{7}$, intriguingly, the majority of neutron scattering measurements have reported a lack of magnetic ordering and the absence of spin waves at low fields in this material [16-18]. In a recent study, sharp spin waves emerged when a magnetic field of $0.5 \mathrm{~T}$ or larger was applied, suggesting that the system transitioned into a conventional state [18]. The possible identification of the low-field state with a quantum spin liquid is 
tantalizing, but progress certainly requires a more detailed understanding of the spin Hamiltonian.

In this article, we present a detailed experimental and theoretical investigation of the excitation spectrum in the high-field state throughout the Brillouin zone. We show that the spectrum is extremely well fit by spin wave theory, and through this fit we unambiguously extract all the microscopic exchange parameters in the spin Hamiltonian (see below). Interestingly, we find that the largest exchange interaction is of the same "frustrated ferromagnetic" Ising type as in spin ice, despite the fact that the $g$-tensor tends to orient magnetic moments primarily normal to the Ising axes. Moreover, spin-flip terms which induce quantum dynamics are comparable to the Ising exchange. This result confirms the picture of $\mathrm{Yb}_{2} \mathrm{Ti}_{2} \mathrm{O}_{7}$ as a strongly quantum magnet, in qualitative agreement with recent studies $[10,19,20]$. Strikingly, we find that the predictions of mean-field theory using these parameters disagree drastically with experiments in zero field, indicating that fluctuations strongly reduce or destroy the classically expected spin order. Taken together, these observations make $\mathrm{Yb}_{2} \mathrm{Ti}_{2} \mathrm{O}_{7}$ a promising candidate for the observation of QSL physics. The precise determination of the microscopic spin interactions sets the stage for a quantitative understanding and test of this proposal.

Time-of-flight neutron scattering measurements were performed on a $7 \mathrm{~g}$ single crystal of $\mathrm{Yb}_{2} \mathrm{Ti}_{2} \mathrm{O}_{7}$, grown via the optical-floating-zone method. Details of the crystal growth were given elsewhere [18,21]. The neutron scattering data was collected at the Disk Chopper Spectrometer at the NIST Center for Neutron Research, using $5 \AA$ incident neutrons. This configuration allowed an energy resolution of $0.09 \mathrm{meV}$. The sample environment consisted of a $11.5 \mathrm{~T}$ vertical-field magnet combined with a dilution insert that achieved a base temperature of $30 \mathrm{mK}$. The scattering plane was chosen to contain the directions [110] and [001]. This is known as the HHL plane, and it contains all of the highest-symmetry directions in a cubic lattice. The vertical direction, along which the magnetic field is applied, is then [110]. The sample was rotated $147^{\circ}$ in $1.5^{\circ}$ steps about the vertical, allowing a threedimensional data set to be acquired, i.e., two components of the wave vector $\mathbf{Q}$ within the scattering plane, and energy transfer. The spin-excitation spectra along several high-symmetry directions within the scattering plane were thereby obtained.

At $30 \mathrm{mK}$, the inelastic spectrum changes qualitatively at $H=0.5 \mathrm{~T}$; above this field strength, resolution-limited spin wave excitations that go soft with quadratic dispersion at nuclear-allowed positions develop, indicating a transition to a field-polarized ferromagnetic state [18]. The spin wave excitations indicate that the symmetry of the underlying lattice is preserved, as is evident from gaps in the spectrum at the nuclear zone boundaries. In Fig. 1 we show the spin wave dispersions along several directions in the HHL plane for $H=2 \mathrm{~T}$ and $H=5 \mathrm{~T}$. These high- symmetry directions are shown relative to the Brillouinzone structure within the HHL plane in Fig. 2.

We compare the experimental data to spin wave theory. We assume nearest-neighbor exchange coupling only, as appropriate to the strongly localized $f$-electron states, and neglect dipolar interactions. The Hamiltonian, written in global spin coordinates, is then

$$
H=\frac{1}{2} \sum_{i j} J_{i j}^{\mu \nu} S_{i}^{\mu} S_{j}^{\nu}-\mu_{B} H^{\mu} \sum_{i} g_{i}^{\mu \nu} S_{i}^{\nu},
$$

where $J_{i j}^{\mu \nu}=J_{j i}^{\nu \mu}$ is the matrix of exchange couplings between sites $i$ and $j, g_{i}^{\mu \nu}$ is the $g$-tensor for the spin at site $i$, and we take $\hbar=1$. Symmetry allows four independent exchange constants [7], $J_{1}, \ldots, J_{4}$. To specify them, we give the exchange matrix on one pair of nearestneighbor sites, located at positions $\mathbf{r}_{0}=\frac{a}{8}(1,1,1)$ and $\mathbf{r}_{1}=$ $\frac{a}{8}(1,-1,-1)$ on a tetrahedron centered at the origin $[a$ is the conventional cubic lattice spacing for the face centered cubic (FCC) Bravais lattice]:

$$
\mathbf{J}_{01}=\left(\begin{array}{ccc}
J_{2} & J_{4} & J_{4} \\
-J_{4} & J_{1} & J_{3} \\
-J_{4} & J_{3} & J_{1}
\end{array}\right) .
$$

The other exchange matrices can be obtained from this one by cubic rotations given in Appendix A. The $g$-tensor contains two components: $g_{z}$ parallel to and $g_{x y}$ perpendicular to the local $C_{3}$ rotation axis through the $\mathrm{Yb}$ site.

Spin wave theory, carried out as described in Appendix $\mathrm{C}$, is fit to the $H=5 \mathrm{~T}, T=30 \mathrm{mK}$ measurements; the fitting procedure focuses on the dispersion relation alone, and the overall intensity of the calculated spin waves is scaled to agree with the experiment at a single wave vector and energy point. The resulting inelastic structure factor $S(\mathbf{Q}, \omega)$ (see Appendix C) is compared to both the $5 \mathrm{~T}$ and $2 \mathrm{~T}$ data in Fig. 1. The best fit is achieved with the following exchange parameters, in $\mathrm{meV}$ :

$$
\begin{array}{ll}
J_{1}=-0.09 \pm 0.03, & J_{2}=-0.22 \pm 0.03, \\
J_{3}=-0.29 \pm 0.02, & J_{4}=0.01 \pm 0.02 .
\end{array}
$$

Here we quote rough uncertainties obtained by the visual comparison of the theoretical and experimental intensities. The fit is performed by taking the ratio of components of the $g$-tensor to be $g_{x y} / g_{z}=2.4$, i.e., the ratio obtained by Ref. [11]. The fit then produces $g_{z}=1.80$, in nearly perfect agreement with these studies (using the $g$-factor ratio of Cao et al. instead [19], i.e., $g_{x y} / g_{z}=1.8$ does not reproduce the data as precisely). Using these results, a high-temperature expansion gives (see Appendix B) a theoretical Curie-Weiss temperature $\Theta_{\mathrm{CW}}=312 \mathrm{mK}$, which is comparable to, but smaller than, the experimentally determined values, $\Theta_{\mathrm{CW}}=$ $400 \mathrm{mK}$ [22] and $750 \mathrm{mK}$ [11]. The deviations may be explained by the sensitivity of the theoretical value to small changes in the $g$-factors and exchange parameters, and to the dependence of the experimental value on the fitting 

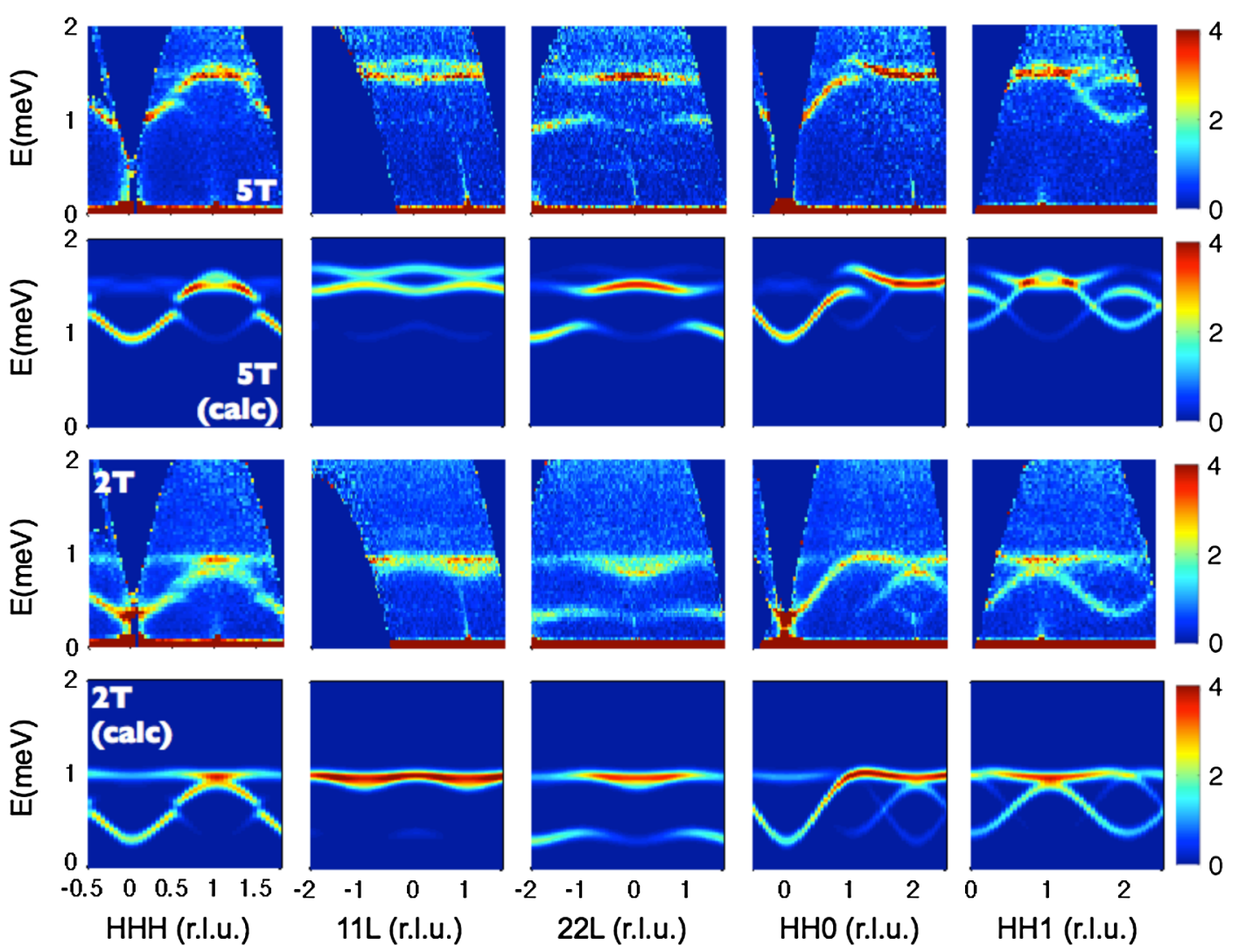

FIG. 1. The measured $S(\mathbf{Q}, \omega)$ at $T=30 \mathrm{mK}$, sliced along various directions in the HHL plane, for both $H=5 \mathrm{~T}$ (first row) and $H=2 \mathrm{~T}$ (third row). The second and fourth rows show the calculated spectrum for these two field strengths, based on an anisotropic exchange model with five free parameters (see text) that were extracted by fitting to the $5 \mathrm{~T}$ data set. For a realistic comparison to the data, the calculated $S(\mathbf{Q}, \omega)$ is convoluted with a Gaussian of full-width 0.09 meV. Both the $2 \mathrm{~T}$ and $5 \mathrm{~T}$ data sets, composed of spin wave dispersions along five different directions, are described extremely well by the same parameters. (Note that r.l.u. stands for reciprocal lattice units.)

range [19]. Furthermore, and most importantly, our extracted exchange parameters correctly reproduce relative intensities as well as the shape of the spin wave dispersion for each of the five directions. Agreement is excellent for $H=2 \mathrm{~T}$, showing that these parameters produce a robust description of the field-induced ferromagnetic state. We note, however, that there is a significant quantitative disagreement with the exchange parameters quoted in Refs. $[9,10]$ (see Appendix H).

Implications.-The excellent agreement with spin wave theory for fields $H \geq 2 \mathrm{~T}$ clearly indicates that the high field state is accurately modeled semiclassically, and is smoothly connected to the fully polarized limit. Theoretically, the ground state in this regime breaks no symmetries, and supports a ferromagnetic polarization along the axis of the applied field (for the $\langle 110\rangle$ field used in the experiment). However, the semiclassical analysis clearly and dramatically fails at small fields, where the measurements show no signs of spontaneous long-range order [18]. The classical zerofield ground state for our Hamiltonian parameters has a large spontaneous polarization along the $\langle 100\rangle$ axis. Extension of this analysis to a $T>0$ mean-field theory wrongly predicts a
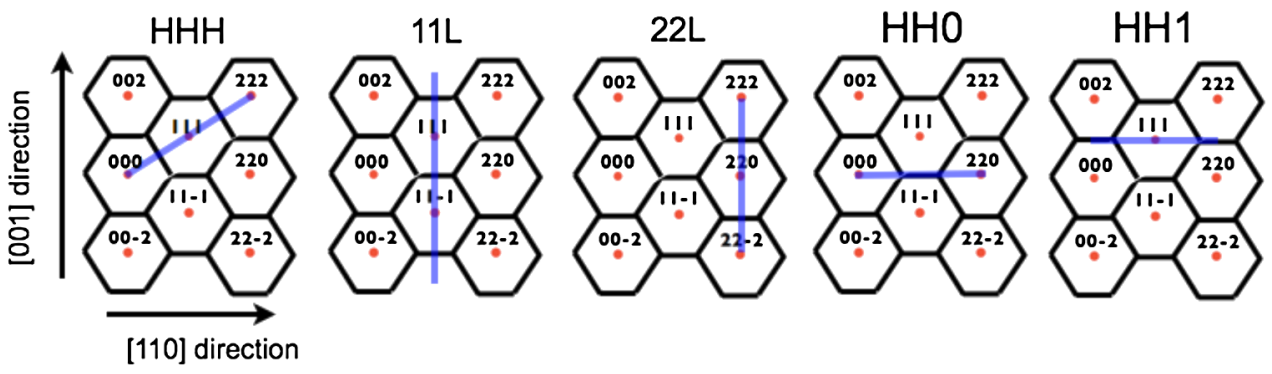

FIG. 2. Representations of the HHL scattering plane, showing the FCC Brillouin-zone boundaries and the corresponding zone centers (labeled in terms of the conventional simple-cubic unit cell). Blue lines indicate the directions of the five different cuts shown in Fig. 1. 
continuous magnetic ordering transition at a temperature of $T_{c}^{\mathrm{MF}}=3.2 \mathrm{~K}$ (see Appendix F). The experimental indications of a zero-field transition to long-range order are mixed $[15,17]$, but early specific heat measurements [22] found an anomaly at $T_{c}=214 \mathrm{mK}$, and Mössbauer spectroscopy [16] suggested a transition at $240 \mathrm{mK}$. This temperature is approximately 14 times lower than $T_{c}^{\mathrm{MF}}$. If there is magnetic ordering at all, it appears to be substantially suppressed, indicating strong fluctuations-classical, quantum, or both.

The presence of strong fluctuations makes a QSL ground state plausible in low fields. We now use the Hamiltonian parameters to suggest the nature of this state. To do so, we rewrite the zero-field Hamiltonian in terms of spins quantized along the local $C_{3}$ axis for each site, similarly to Ref. [20]:

$$
\begin{aligned}
H= & \sum_{\langle i j\rangle}\left\{J_{z z} \mathrm{~S}_{i}^{z} \mathrm{~S}_{j}^{z}-J_{ \pm}\left(\mathrm{S}_{i}^{+} \mathrm{S}_{j}^{-}+\mathrm{S}_{i}^{-} \mathrm{S}_{j}^{+}\right)\right. \\
& +J_{ \pm \pm}\left(\gamma_{i j} \mathrm{~S}_{i}^{+} \mathrm{S}_{j}^{+}+\gamma_{i j}^{*} \mathrm{~S}_{i}^{-} \mathrm{S}_{j}^{-}\right) \\
& \left.+J_{z \pm}\left[\mathrm{S}_{i}^{z}\left(\zeta_{i j} \mathrm{~S}_{j}^{+}+\zeta_{i j}^{*} \mathrm{~S}_{j}^{-}\right)+i \leftrightarrow j\right]\right\},
\end{aligned}
$$

where here $\mathrm{S}_{i}^{\mu}$ are local spin coordinates, $J_{z z}=$ $-\frac{1}{3}\left[2 J_{1}-J_{2}+2\left(J_{3}+2 J_{4}\right)\right], \quad J_{ \pm}=\frac{1}{6}\left(2 J_{1}-J_{2}-J_{3}-\right.$ $\left.2 J_{4}\right), J_{ \pm \pm}=\frac{1}{6}\left(J_{1}+J_{2}-2 J_{3}+2 J_{4}\right)$, and $J_{z \pm}=\frac{1}{3 \sqrt{2}}\left(J_{1}+\right.$ $\left.J_{2}+J_{3}-J_{4}\right)$, and the matrices $\gamma_{i j}, \zeta_{i j}$ consist of unimodular complex numbers (given in Appendix A). From the fits in Eq. (3), we find, in meV,

$$
\begin{aligned}
& J_{z z}=0.17 \pm 0.04, \quad J_{ \pm}=0.05 \pm 0.01, \\
& J_{ \pm \pm}=0.05 \pm 0.01, \quad J_{z \pm}=-0.14 \pm 0.01,
\end{aligned}
$$

where the uncertainties have been estimated by treating those in Eq. (3) as Gaussian random variables. Note that the strongest interaction is $J_{z z}>0$, which precisely coincides with the nearest-neighbor spin ice model. The model with only $J_{ \pm}$and $J_{z z}$ has been studied theoretically $[13,23]$. It does indeed support a QSL ground state for sufficiently small $J_{ \pm} / J_{z z}$. For larger $J_{ \pm} / J_{z z}$, the ground state is instead a magnet with $\left\langle S_{i}^{ \pm}\right\rangle \neq 0$ [23]. While the actual value of $J_{ \pm} / J_{z z} \approx 0.3$ would place this model in the magnetic state [23], the $J_{z \pm}$ interaction, in particular, is non-negligible in $\mathrm{Yb}_{2} \mathrm{Ti}_{2} \mathrm{O}_{7}$, and preliminary theoretical work suggests that it tends to stabilize the QSL state. Indeed, in perturbation theory, the leading effect of the $J_{z \pm}$ coupling is to induce, in the effective Hamiltonian, a term close to the RokhsarKivelson interaction of Ref. [13] (see Appendix G), which was shown to stabilize the QSL $[13,24]$. Although perturbation theory is, strictly speaking, only valid for $J_{z \pm} / J_{z z} \ll 1$, the conclusions of this analysis are likely to extend to a larger range of values. A nonperturbative study of the full Hamiltonian in Eq. (4) is beyond the scope of this paper, but will be reported in a future publication. Given the uncertainty in the phase boundary for the QSL state, we cannot disregard the possibility that an ideal sample of $\mathrm{Yb}_{2} \mathrm{Ti}_{2} \mathrm{O}_{7}$ would be magnetically ordered, but that such order is here disrupted by crystal defects, inducing, for example, the formation of nanoscale domain walls. This possibility should be pursued further in the future, taking into account the new understanding of the Hamiltonian. We proceed now to discuss the implications of the alternative possibility of a zero-field QSL state.

Many of the key properties of the QSL state of the $J_{z z}-$ $J_{ \pm}$model were established in Ref. [13]. Conceptually, it is a quantum analog of the classical regime of Coulombic correlations observed in spin ice [5]. Specifically, where spin ice realizes an analog of magnetostatics, the QSL state of Eq. (4) embodies a complete fictitious quantum electrodynamics. In this phase, the magnetic monopoles of spin ice become full-fledged coherent excitations of the system. In addition, the QSL supports dual electric monopoles and a dynamical emergent photon mode at low energies. The complex and largely diffuse character of the scattering in zero field [18] may well be a consequence of the combination of these diverse excitations. Indeed, where a neutron can create just one spin wave $(S=1)$ excitation, the $S=$ $1 / 2$ magnetic monopoles are excited in pairs with no individual momentum-conservation constraints. A careful

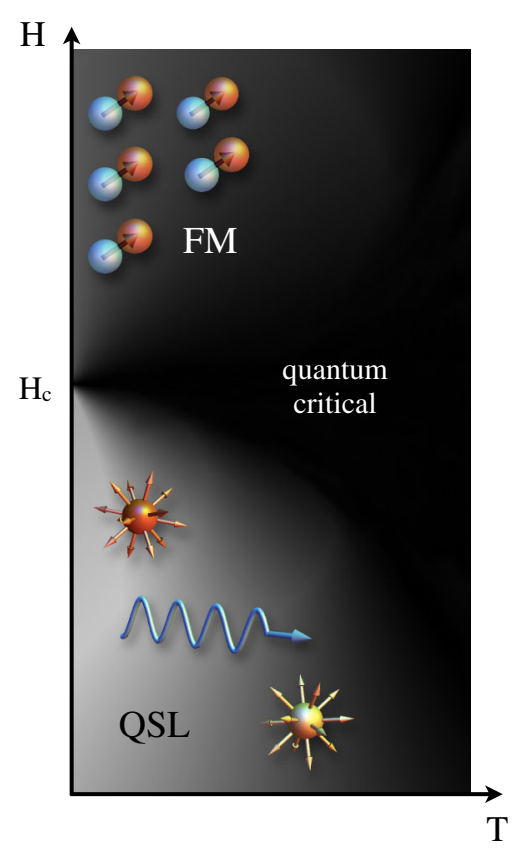

FIG. 3. Schematic phase diagram in the temperature $(T)$ magnetic-field $(H)$ plane, for a material in the QSL phase of Eq. (1) at $T=H=0$. At a low field and temperature, the QSL state supports exotic excitations: "magnetic" (red sphere) and "electric" (yellow sphere) monopoles, and an emergent photon (wavy line). The field $H_{c}$ marks a quantum critical point: the confinement phase transition. For $H>H_{c}$, the ground state is a simple field-polarized ferromagnet (FM), and the elementary excitations are conventional magnetic dipoles. The gradations in gray scale indicate crossovers to the quantum critical region between them, governed by the $T=0$ confinement phase transition. 
comparison of theoretical modeling and focused experiments in low fields are clearly needed.

A key consequence of the QSL scenario is the presence of a quantum confinement phase transition in applied field (see Fig. 3). Such a quantum phase transition is required to remove the "fractional" excitations of the QSL phase (electric and magnetic monopoles) from the spectrum in the semiclassical high-field phase. Theoretically, such quantum critical points have been studied in related model Hamiltonians, and occur by a mechanism analogous to the Higgs transition in the standard model [25] or by magnetic monopole condensation [26]. The gapless excitations observed by neutrons at $H \approx 0.5 \mathrm{~T}$ in Ref. [18] indeed indicate a quantum critical point at this field. Further theoretical work is required for a detailed comparison to experiment.

$\mathrm{Yb}_{2} \mathrm{Ti}_{2} \mathrm{O}_{7}$ enjoys several major advantages over other materials considered to be candidates for QSL states up to now [14]: It is the only case in which the Hamiltonian parameters are precisely known, and for which large single-crystal samples highly suitable for detailed neutron scattering measurements are available. Moreover, similar methods may be applicable to other rare-earth pyrochlores in which substantial quantum effects are present, such as $\mathrm{Er}_{2} \mathrm{Ti}_{2} \mathrm{O}_{7}$ and $\mathrm{Tb}_{2} \mathrm{Ti}_{2} \mathrm{O}_{7}[1,8]$. Especially in this broader context, the prospects for detailed observation of the longsought QSL physics are bright. The basic framework established here will allow coordinated theoretical and experimental studies to confront the problem.

We thank M. Gingras for abundant comments on a first draft. K. A.R. acknowledges useful discussions with B. Prasanna Venkatesh. L. S. acknowledges B. Canals and P. Holdsworth for many enlightening discussions, and the Institut Néel, Grenoble for hospitality. K. A. R. and B.D. G. were supported by NSERC of Canada. L. B. and L. S. were supported by the DOE through Basic Energy Sciences Grant No. DE-FG02-08ER46524.

\section{APPENDIX A: CUBIC ROTATIONS AND LOCAL BASES}

As described in the main text, we use the usual coordinate system for the pyrochlore lattice, with sites located on tetrahedra whose centers form a FCC lattice. We take one to be centered at the origin with its four corners at $\mathbf{r}_{0}=$ $\frac{a}{8}(1,1,1), \mathbf{r}_{1}=\frac{a}{8}(1,-1,-1), \mathbf{r}_{2}=\frac{a}{8}(-1,1,-1)$, and $\mathbf{r}_{3}=$ $\frac{a}{8}(-1,-1,1)$. The exchange matrices $\mathbf{J}_{i j}$ between sites of types $i$ and $j$ are obtained by applying the following cubic rotations $\mathcal{R}_{i j}$ to $\mathbf{J}_{01}$ :

(i) $\mathcal{R}_{02}$ is a $\frac{2 \pi}{3}$ rotation about the [111] axis,

(ii) $\mathcal{R}_{03}$ is a $\frac{4 \pi}{3}$ rotation about the [111] axis,

(iii) $\mathcal{R}_{21}$ is a $\frac{4 \pi}{3}$ rotation about the $[1 \overline{1} \overline{1}]$ axis,

(iv) $\mathcal{R}_{31}$ is a $\frac{2 \pi}{3}$ rotation about the $[1 \overline{1} \overline{1}]$ axis,

(v) $\mathcal{R}_{23}$ is a rotation made of a $\frac{2 \pi}{3}$ rotation about the [111] axis followed by a $\frac{4 \pi}{3}$ rotation about the [1 $\left.1 \overline{1} \overline{1}\right]$ axis.
Note $\mathbf{J}_{j i}=\mathbf{J}_{i j}^{T}$.

We use the following local $\left(\hat{\mathbf{a}}_{i}, \hat{\mathbf{b}}_{i}, \hat{\mathbf{e}}_{i}\right)$ bases:

$\hat{\mathbf{e}}_{0}=\frac{1}{\sqrt{3}}(1,1,1), \quad \hat{\mathbf{a}}_{0}=\frac{1}{\sqrt{6}}(-2,1,1)$,

$\hat{\mathbf{e}}_{1}=\frac{1}{\sqrt{3}}(1,-1,-1), \quad \hat{\mathbf{a}}_{1}=\frac{1}{\sqrt{6}}(-2,-1,-1)$,

$\hat{\mathbf{e}}_{2}=\frac{1}{\sqrt{3}}(-1,1,-1), \quad \hat{\mathbf{a}}_{2}=\frac{1}{\sqrt{6}}(2,1,-1)$,

$\hat{\mathbf{e}}_{3}=\frac{1}{\sqrt{3}}(-1,-1,1), \quad \hat{\mathbf{a}}_{3}=\frac{1}{\sqrt{6}}(2,-1,1)$,

$\hat{\mathbf{b}}_{i}=\hat{\mathbf{e}}_{i} \times \hat{\mathbf{a}}_{i}$, and the $4 \times 4$ complex unimodular matrices

$\zeta=\left(\begin{array}{cccc}0 & -1 & e^{i \pi / 3} & e^{-i \pi / 3} \\ -1 & 0 & e^{-i \pi / 3} & e^{i \pi / 3} \\ e^{i \pi / 3} & e^{-i \pi / 3} & 0 & -1 \\ e^{-i \pi / 3} & e^{i \pi / 3} & -1 & 0\end{array}\right), \quad \gamma=-\zeta^{*}$,

for which our exchange Hamiltonian takes the form of Eq. (4).

\section{APPENDIX B: CURIE-WEISS TEMPERATURE}

A high-temperature expansion of the Hamiltonian of Eq. (1) yields the $O\left(1 / T^{2}\right)$ term in the uniform susceptibility from which we extract the Curie-Weiss temperature,

$$
\begin{aligned}
\Theta_{\mathrm{CW}}= & \frac{-1}{6 k_{B}\left(2 g_{x y}^{2}+g_{z}^{2}\right)}\left[2 g_{x y}^{2}\left(4 J_{1}+J_{2}-5 J_{3}+2 J_{4}\right)\right. \\
& +8 g_{x y} g_{z}\left(J_{1}+J_{2}+J_{3}-J_{4}\right) \\
& \left.+g_{z}^{2}\left(2 J_{1}-J_{2}+2 J_{3}+4 J_{4}\right)\right],
\end{aligned}
$$

where $k_{B}$ is the Boltzmann constant. Using the formulation of Eq. (4) $\Theta_{\mathrm{CW}}$ takes the simpler form

$$
\begin{aligned}
\Theta_{\mathrm{CW}}= & \frac{1}{2 k_{B}\left(2 g_{x y}^{2}+g_{z}^{2}\right)}\left[g_{z}^{2} J_{z z}-4 g_{x y}^{2}\left(J_{ \pm}+2 J_{ \pm \pm \pm}\right)\right. \\
& \left.-8 \sqrt{2} g_{x y} g_{z} J_{z \pm}\right] .
\end{aligned}
$$

\section{APPENDIX C: SPIN WAVE THEORY}

As usual, we expand the Hamiltonian about one of the classical states using Holstein-Primakoff bosons in the spirit of large $s$, and keep only terms up to and of order $s$, which shall then be set to $1 / 2$. We define the transverse Holstein-Primakoff bosons $x_{a}=x_{a}^{\dagger}, y_{a}=y_{a}^{\dagger}$, conjugate with one another on site $a$ of the pyrochlore lattice, satisfying

$$
\left[x_{a}, y_{a}\right]=i, \quad n_{a}=\frac{x_{a}^{2}+y_{a}^{2}}{2}-\frac{1}{2},
$$

such that 


$$
\begin{gathered}
\mathbf{S}_{a} \cdot \mathbf{u}_{a}=s-n_{a}, \quad \mathbf{S}_{a} \cdot \mathbf{v}_{a}=\sqrt{s} x_{a}, \\
\mathbf{S}_{a} \cdot \mathbf{w}_{a}=\sqrt{s} y_{a} .
\end{gathered}
$$

Here $\left(\mathbf{v}_{a}, \mathbf{w}_{a}, \mathbf{u}_{a}\right)$ is an orthonormal basis, chosen such that $\mathbf{u}_{a}$ gives the direction of the spin in the classical ground state at site $a$ (which we find numerically). We find that for all fields, the ground state does not enlarge the unit cell, so that there are only four distinct such bases, which we denote by $a=0, \ldots, 3$. One may choose, for example, $\mathbf{v}_{a}=\mathbf{u}_{a} \times(1,1,1) /\left\|\mathbf{u}_{a} \times(1,1,1)\right\|$ and $\mathbf{w}_{a}=\mathbf{u}_{a} \times \mathbf{v}_{a}$.

Since the classical ground state does not enlarge the unit cell, we can readily proceed to Fourier space in the four site basis. Keeping only terms of order $s$, we arrive at the spin wave (quadratic) Hamiltonian,

$$
H_{\mathbf{k}}=\left(\begin{array}{ll}
X_{-\mathbf{k}}^{T} & Y_{-\mathbf{k}}^{T}
\end{array}\right)\left(\begin{array}{cc}
A_{\mathbf{k}} & C_{\mathbf{k}} \\
C_{\mathbf{k}}^{T} & B_{\mathbf{k}}
\end{array}\right)\left(\begin{array}{c}
X_{\mathbf{k}} \\
Y_{\mathbf{k}}
\end{array}\right),
$$

where $\left(X^{T} Y^{T}\right)=\left(x_{0}, \ldots, x_{3}, y_{0}, \ldots, y_{3}\right) \quad$ and $\quad D_{\mathbf{k}}^{a b}=$ $\tilde{D}_{a b} \cos \left[\mathbf{k} \cdot\left(\mathbf{r}_{a}-\mathbf{r}_{b}\right)\right], D=A, B, C$, with

$$
\begin{aligned}
\tilde{A}_{a b}= & s \mathbf{v}_{a} \cdot \mathbf{J}_{a b} \cdot \mathbf{v}_{b}+\frac{\mu_{B}}{2} \mathbf{H} \cdot \mathbf{g}_{a} \cdot \mathbf{u}_{a} \delta_{a b} \\
& -s \mathbf{u}_{a} \cdot \sum_{b} \mathbf{J}_{a b} \cdot \mathbf{u}_{b}, \\
\tilde{B}_{a b}= & s \mathbf{w}_{a} \cdot \mathbf{J}_{a b} \cdot \mathbf{w}_{b}+\frac{\mu_{B}}{2} \mathbf{H} \cdot \mathbf{g}_{a} \cdot \mathbf{u}_{a} \delta_{a b} \\
& -s \mathbf{u}_{a} \cdot \sum_{b} \mathbf{J}_{a b} \cdot \mathbf{u}_{b}, \\
\tilde{C}_{a b}= & s \mathbf{v}_{a} \cdot \mathbf{J}_{a b} \cdot \mathbf{w}_{b},
\end{aligned}
$$

where $\mathbf{H}$ is the magnetic field and $\mathbf{J}_{a b}$ and $\mathbf{g}_{a}$ are $3 \times 3$ matrices with matrix elements $J_{a b}^{\mu \nu}$ and $g_{a}^{\mu \nu}$, respectively. To find the modes, we resort to the path-integral formulation. The action at temperature $T=1 /\left(k_{B} \beta\right)$ is

$$
\mathcal{S}=\frac{1}{2 \beta} \sum_{n} \sum_{\mathbf{k}} Z_{-\mathbf{k},-\omega_{n}}^{T}\left[G_{\mathbf{k}}+\left(i \omega_{n}\right) \Gamma\right] Z_{\mathbf{k}, \omega_{n}},
$$

where $\omega_{n}=\frac{2 \pi n}{\beta}$ is the Matsubara frequency, where we have defined $Z^{T}=\left(\begin{array}{ll}X^{T} & Y^{T}\end{array}\right)$,

$$
G_{\mathbf{k}}=2\left(\begin{array}{cc}
A_{\mathbf{k}} & C_{\mathbf{k}} \\
C_{\mathbf{k}}^{T} & B_{\mathbf{k}}
\end{array}\right) \text { and } \Gamma=\left(\begin{array}{cc}
0 & -i \mathbf{1}_{4} \\
i \mathbf{1}_{4} & 0
\end{array}\right)
$$

$\left(\mathbf{1}_{4}\right.$ is the four-by-four identity matrix). As usual, the real frequency dispersion relations $\omega(\mathbf{k})$ are found by solving the zero-eigenvalue equations of the matrix $G_{\mathbf{k}}+\omega \Gamma$. Here, these are equivalently the (both zero and nonzero) eigenvalues of $-\Gamma G_{\mathbf{k}}$.

We calculate the inelastic structure factor (to which the intensity $I(\mathbf{k}, \omega)$ of the scattering is proportional) obtained from the moment-moment correlation function [27],
$S(\mathbf{k}, \omega)$
$=\sum_{\mu, \nu}\left[\delta_{\mu \nu}-(\hat{\mathbf{k}})_{\mu}(\hat{\mathbf{k}})_{\nu}\right] \sum_{a, b}\left\langle m_{a}^{\mu}(-\mathbf{k},-\omega) m_{b}^{\nu}(\mathbf{k}, \omega)\right\rangle$,

where $\hat{\mathbf{k}}$ is the unit vector associated with $\mathbf{k}, m_{a}^{\mu}(\mathbf{k}, \omega)=$ $\mu_{B} \sum_{\kappa} g_{a}^{\mu \kappa} S_{a}^{\kappa}(\mathbf{k}, \omega)$ is the moment at momentum $\mathbf{k}$ and real frequency $\omega$ on the $a=0, \ldots, 3$ sublattice in direction $\mu=x, y, z$, and $S_{a}^{\kappa}$ is, as usual, the $\kappa$-th coordinate of the effective spin-1/2 spin at site $a . \mathcal{F}_{\mu \nu}(\mathbf{k})=$ $\delta_{\mu \nu}-(\hat{\mathbf{k}})_{\mu}(\hat{\mathbf{k}})_{\nu}$ selects the component of the spin-spin correlations perpendicular to the scattering vector [27], and $\quad G_{a b}^{\mu \nu}(\mathbf{k}, \omega)=\left\langle m_{a}^{\mu}(-\mathbf{k},-\omega) m_{b}^{\nu}(\mathbf{k}, \omega)\right\rangle \quad$ is the moment-moment correlation function that originates from the interaction between the neutron's moment and the spins' moments, which we find to be

$G_{a b}^{\mu \nu}$

$=-\pi s \eta_{a}^{\mu} \eta_{b}^{\nu} \sum_{\alpha} \delta\left(\omega-\epsilon_{\alpha}\right) \frac{1}{\psi_{R, \alpha}^{\dagger} \Gamma \psi_{R, \alpha}}\left(\left[\psi_{R, \alpha}^{\dagger}\right]_{a}\left[\psi_{R, \alpha}\right]_{b}\right)$,

where $\delta$ is the Dirac distribution, $\eta_{a}^{\mu}=$ $\sum_{\kappa=x, y, z} g_{a}^{\mu \kappa}\left(v_{a}^{\kappa} w_{a}^{\kappa}\right), \epsilon_{\alpha}$ is the $\alpha$-th eigenvalue of $-\Gamma G$, and $\psi_{R, \alpha}$ is its corresponding "right" eigenvector, i.e., such that $-\Gamma G \cdot \psi_{R, \alpha}=\epsilon_{\alpha} \psi_{R, \alpha}$. Also note that the momentum and frequency dependencies are implied everywhere to be $\mathbf{k}, \omega$.

Now we estimate the amplitude of quantum fluctuations using our spin wave theory, by evaluating the quantum moment reduction in zero field and at zero temperature. In the Holstein-Primakoff boson language the reduction of the spin expectation value from the classical value of $1 / 2$, averaged over the four site basis, is $r=\frac{1}{4} \sum_{a=0}^{3}\left\langle 0\left|n_{a}\right| 0\right\rangle$, where $\left\langle 0\left|n_{a}\right| 0\right\rangle$ indicates a ground-state quantum expectation value. Evaluating this using the path-integral method, we obtain $r=\frac{1}{8} \int_{\mathbf{k}, \omega} \operatorname{Tr}\left\langle\left[G_{\mathbf{k}}+\omega \Gamma\right]^{-1}\right\rangle-\frac{1}{2}$, i.e.,

$$
r=\frac{1}{8} \int_{\mathbf{k}} \sum_{\alpha} \Theta\left(\epsilon_{\alpha}\right) \frac{\psi_{R, \alpha}^{\dagger} \psi_{R, \alpha}}{\psi_{R, \alpha}^{\dagger} \Gamma \psi_{R, \alpha}}-\frac{1}{2} \approx 0.05
$$

$(\Theta$ is the usual Heaviside distribution), which is a $10 \%$ reduction compared with the classical value of $1 / 2$.

\section{APPENDIX D: FURTHER DETAILS OF EXPERIMENTAL DATA AND FITS}

The inelastic neutron scattering data (rows 1 and 3 in Fig. 1) contain several features worth commenting on further. First, the darkest blue areas do not contain any data, either for kinematic reasons near $\mathbf{Q}=(0,0,0)$, or because of the finite angular extend of the scan. Second, at $E=0$ one observes intensity that is due to coherent and incoherent elastic scattering from the sample, and hence is 

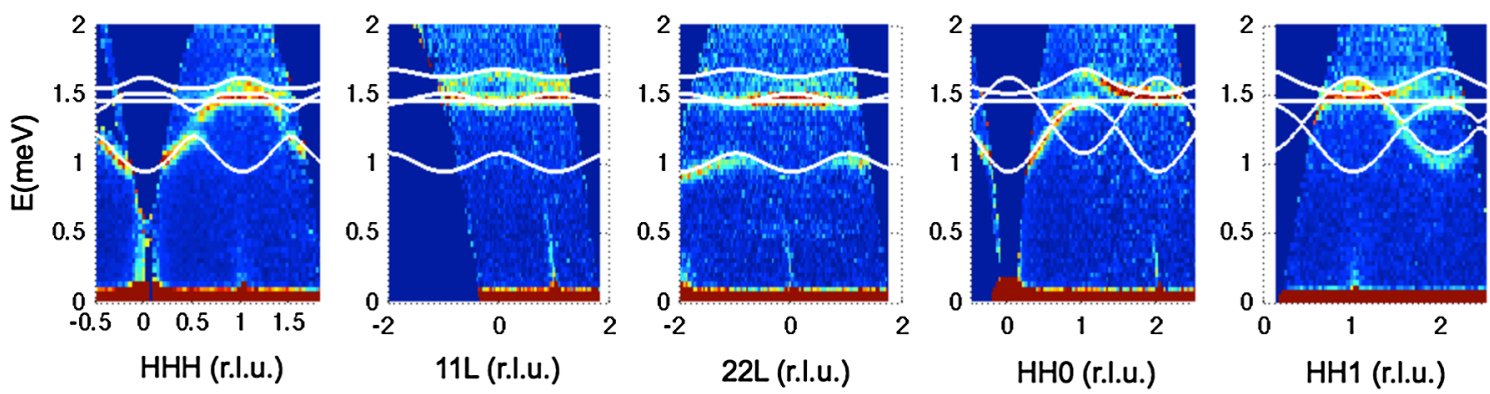

FIG. 4. Dispersions (white curves) obtained from the fitting procedure over-plotted on the data at $H=5 \mathrm{~T}$.

more intense than the inelastic features by up to several orders of magnitude, thus appearing red (off scale). Third, near the $(0,0,0)$ position there is higher background leading to unphysical intensities due to contamination from the unscattered incident beam. This is observable in the $\mathrm{HHH}$ and HHO data sets near the zero position.

The data sets were collected by counting for 8 minutes per angular rotation of the sample. The total time per magnetic-field setting was about 12 hours.

Figure 4 shows the dispersions obtained from the fitting procedure over-plotted on the data. These fits were accomplished by digitizing the shape of the dispersion from the experimental data, and performing a least squares minimization routine to match it. Intensities were calculated based on the spin wave theory using the extracted exchange parameters, and were not fit to the data. The 5D parameter space (four exchange plus one $g$-tensor parameter) was explored using a uniform search technique from which the resulting excellent description was obtained (Fig. 1).

\section{APPENDIX E: FEATURES OF THE SPIN WAVE SPECTRUM}

The spectra of Fig. 1 are very rich. Because of the many parameters and of the presence of classical phase transitions (there are several ground states to the classical model), it is very difficult to track which features are due to what terms of Eq. (4). One remarkable feature which can be easily identified throughout most of phase space (including the region around which Eq. (3) places $\mathrm{Yb}_{2} \mathrm{Ti}_{2} \mathrm{O}_{7}$ ) is a quasiflat band. Specifically, one spin wave mode is completely dispersionless in the plane of reciprocal space with $k_{x}=k_{y}$, i.e., normal to the magnetic-field direction and passing through the origin in reciprocal space. All scattering measurements on $\mathrm{Yb}_{2} \mathrm{Ti}_{2} \mathrm{O}_{7}$ have been taken in this plane, so this feature is quite significant in the experiments. In the region of phase space around $\mathrm{Yb}_{2} \mathrm{Ti}_{2} \mathrm{O}_{7}$ and for $H=5 \mathrm{~T}$, we find its energy to be, numerically, in meV:

$$
E_{2 \mathrm{D} \mathrm{flat}} \approx 0.74+0.51 J_{z z}-1.18 J_{ \pm}-3.11 J_{ \pm \pm \pm}-5.81 J_{z \pm},
$$

where the $J_{\alpha}$ 's must be input in $\mathrm{meV}$, and which for our fit gives $E_{2 \mathrm{D} \text { flat }}^{\mathrm{Yb}_{2} \mathrm{Ti}_{2} \mathrm{O}_{7}} \approx 1.45 \mathrm{meV}$. Note that the energy of this feature is most sensitive to $J_{z \pm \pm}$.
Moreover, we observe the following trends in the region around $\mathrm{Yb}_{2} \mathrm{Ti}_{2} \mathrm{O}_{7}$ :

(i) as $\left|J_{z \pm}\right|$ increases, all bands go up in energy (especially the two-dimensional flat one),

(ii) increases in $J_{ \pm}$and $J_{ \pm \pm}$seem to have more or less the same effect: The bands get closer, and this happens, in particular, because the energy of the top bands decreases.

\section{APPENDIX F: MEAN-FIELD-THEORY CALCULATION}

The Curie-Weiss mean-field Hamiltonian obtained from Eq. (1) takes the form

$$
\begin{aligned}
H_{\mathrm{MF}}= & \sum_{\langle i, j\rangle} \sum_{\mu, \nu} J_{i j}^{\mu \nu}\left(\left\langle S_{i}^{\mu}\right\rangle S_{j}^{\nu}+S_{i}^{\mu}\left\langle S_{j}^{\nu}\right\rangle-\left\langle S_{i}^{\mu}\right\rangle\left\langle S_{j}^{\nu}\right\rangle\right) \\
& -\mu_{B} H^{\mu} \sum_{i} g_{i}^{\mu \nu} S_{i}^{\nu},
\end{aligned}
$$

where $H^{\mu}$ is the magnetic field in the $\mu$ direction, $\left\langle S_{i}^{\mu}\right\rangle$ is the mean-field quantum thermal expectation value of $S_{i}^{\mu}$, defined by $\left\langle S_{i}^{\mu}\right\rangle=\frac{1}{Z} \operatorname{Tr} S_{i}^{\mu} \exp \left(-\beta H_{\mathrm{MF}}\right)$, where $\beta$ is the inverse temperature $\beta=1 /\left(k_{B} T\right), k_{B}$ is the Boltzmann constant, and $Z$ is the partition function $Z=$ $\operatorname{Tr} \exp \left(-\beta H_{\mathrm{MF}}\right)$. The traces are taken over the up and down spin states of every spin.

The ground state does not enlarge the unit cell at zero temperature, and we assume that this is still the case at nonzero temperature. Thus, we define, for every sublattice $a$ and every axis $\mu$, the average magnetization

$$
m_{a}^{\mu}=\left\langle S_{t, a}^{\mu}\right\rangle,
$$

which is the same for every tetrahedron $t$ ( $t$ can be either "up" or "down"). We arrive at the 12 consistency equations

$$
\mathbf{m}_{a}=-\frac{\mathbf{h}_{a}^{\mathrm{eff}}}{2\left\|\mathbf{h}_{a}^{\mathrm{eff}}\right\|} \tanh \frac{\beta\left\|\mathbf{h}_{a}^{\mathrm{eff}}\right\|}{2},
$$

where $\mathbf{h}_{a}^{\text {eff }}=2 \sum_{b} \mathbf{m}_{b} \cdot \mathbf{J}_{b a}-\mu_{B} \mathbf{H} \cdot \mathbf{g}_{a}$; the free energy per site is 


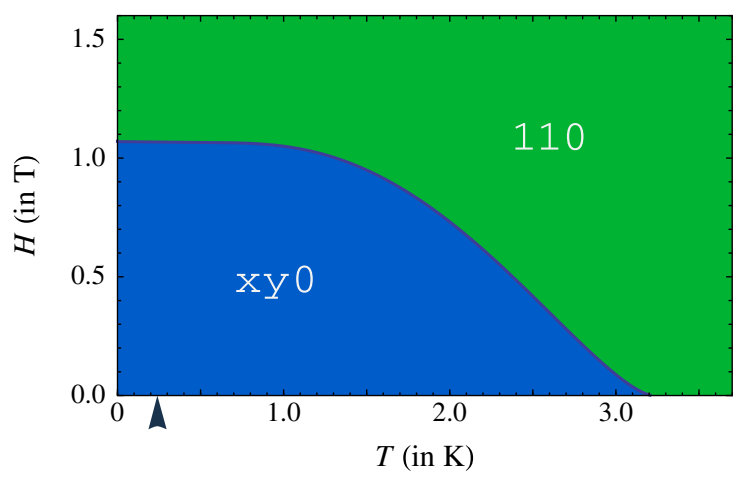

FIG. 5. Field versus temperature phase diagram obtained from a mean-field analysis of the Hamiltonian of Eq. (1), for a field $\mathbf{H}$ parallel to the [110] direction and with the exchange constant values obtained with our fits, Eq. (3). The system displays a net magnetic moment throughout the $(H, T)$ plane. The blue region denotes a region where the total magnetization lies in the $x y$ plane, and the green region is the paramagnetic phase; the two zones are separated by a continuous transition. In zero field, this transition takes place at $T_{c}^{\mathrm{MF}}=3.2 \mathrm{~K}$. At zero temperature, the amplitude of the transition field is $H_{c}^{\mathrm{MF}}=1.1 \mathrm{~T}$. The dark blue arrow shows the experimentally reported transition temperature of $240 \mathrm{mK}$ : The actual transition occurs at a much lower temperature than that predicted by mean-field theory $T_{c}^{\mathrm{MF}}$.

$$
f=-\frac{1}{4} \sum_{a, b} \mathbf{m}_{a} \cdot \mathbf{J}_{a b} \cdot \mathbf{m}_{b}-\frac{1}{4 \beta} \sum_{a} \ln \left[2 \cosh \frac{\beta\left\|\mathbf{h}_{a}^{\mathrm{eff}}\right\|}{2}\right]
$$

We solve Eqs. (F3) numerically, choosing the solution which minimizes the free energy in Eq. (F4). With the field oriented along the [110] direction, we find the phase diagram shown in Fig. 5, which contains two phases. In zero field, the low temperature phase is a ferromagnet with net magnetization along any of the $\langle 100\rangle$ axes. In low fields, this expands into a phase in which the average magnetization is not aligned with the field, but lies within a $\{001\}$ plane. Upon increasing fields, a transition occurs to the high-field state in which the net magnetization is aligned with the applied field, and no symmetries are spontaneously broken. This state is continuously connected to the high-temperature paramagnetic phase.

\section{APPENDIX G: PERTURBATION THEORY}

We show that the $U(1)$ QSL described in Refs. [13,23] is stable to the addition of the terms in the symmetryobtained Hamiltonian, Eq. (4), provided all coupling constants are small with respect to the Ising exchange parameter $J_{z z}$. In other words, we show that the $U(1)$ QSL exists in a finite region of parameter space, specifically where $J_{ \pm}$, $J_{z \pm}$, and $J_{ \pm \pm}$are small with respect to $J_{z z}$.

In that limit, one may apply perturbation theory. When $J_{z z}>0$, the ground-state manifold of the unperturbed Hamiltonian is the extensively degenerate "two-in-twoout" manifold. When $J_{z \pm}=J_{ \pm \pm}=0$, Eq. (4) can be mapped exactly onto the Hamiltonian of Ref. [13], where the first nonvanishing and nonconstant term in perturbation theory (above the "two-in-two-out" manifold) was shown to be third order in $J_{ \pm} / J_{z z}$ :

$$
H_{\text {ring }}^{\mathrm{eff}}=-K \sum_{\{i, j, k, l, m, n\}=0}\left(\mathrm{~S}_{i}^{+} \mathrm{S}_{j}^{-} \mathrm{S}_{k}^{+} \mathrm{S}_{l}^{-} \mathrm{S}_{m}^{+} \mathrm{S}_{n}^{-}+\text {H.c. }\right) \text {, }
$$

where $K=\frac{12 J_{ \pm}^{3}}{J_{2 z}^{2}}$ is a ring exchange interaction. $H_{\text {ring }}^{\text {eff }}$ flips the spins on the "flippable" hexagons, i.e., those with alternating up and down spins, and yields zero otherwise. This represents a ring move responsible for favoring the quantum superpositions of the $U(1)$ QSL. As shown in Refs. [13,23,28], this ring Hamiltonian has as its ground state a $U(1)$ QSL, whose low-energy physics is described as the Coulomb phase of a $U(1)$ gauge theory. This phase is locally stable to all perturbations [13] in three dimensions, which is enough already to guarantee the persistence of the QSL state when the other exchange couplings are sufficiently small, i.e., when the induced terms in the effective Hamiltonian are much smaller than the ring coupling $K$.

We can, however, go further and consider these effects explicitly in the perturbative limit, which extends the discussion to the case when $J_{z \pm}, J_{ \pm \pm} \ll J_{z z}$ but with no particular assumptions placed upon the magnitude of the induced terms in the effective Hamiltonian relative to $K$. When $J_{z \pm}$ or $J_{ \pm \pm}$are nonzero, other "nonring" effective Hamiltonians are allowed. In particular, the $J_{z \pm}$ term gives rise to an effective third-neighbor ferromagnetic Ising Hamiltonian:

$$
H_{3 \mathrm{rd} \text { Ising }}^{\mathrm{eff}}=-J_{(3)} \sum_{\langle\langle i, j\rangle\rangle\rangle} \mathrm{S}_{i}^{z} \mathrm{~S}_{j}^{z}
$$

where $J_{(3)}=\frac{3 J_{z \pm}^{2}}{J_{z z}}$. This term alone has six symmetryrelated ordered ground states. Each of them consists of the choice of one of the six two-in-two-out tetrahedra, with the same pattern repeated on each "up" tetrahedron (these states are magnetic with their moment along the $\langle 100\rangle$ directions) and contain no flippable hexagons; see Fig. 6.

This implies that states which contain flippable hexagons represent an energy cost. We can therefore consider
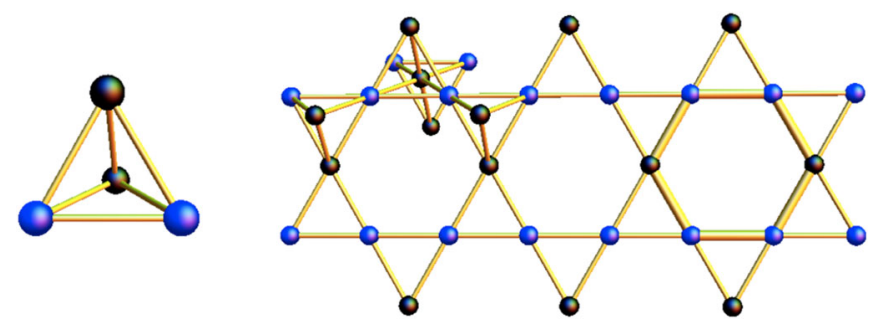

FIG. 6. One of the six ground states of $H_{3 \mathrm{rd} \text { Ising }}^{\text {eff }}=$ $-\frac{3 J_{z \pm}^{2}}{J_{z z}} \sum_{\langle\langle i, j\rangle\rangle)} \boldsymbol{S}_{i}^{z} \boldsymbol{S}_{j}^{z}$. A blue sphere represents an "in" spin while a black sphere represents an "out" one. Because two of the four chain types contain nonalternating spins, there are no flippable hexagons in any of the six (equivalent) ground states. 
$H_{3 \text { rdlsing }}^{\text {eff }}$ as an analog of the Rokhsar-Kivelson (RK) term [24] introduced by Hermele et al. [13], $H_{\mathrm{RK}}=V N_{f}$, where $N_{f}$ is the operator that counts the number of flippable hexagons. This term actually stabilizes the QSL state [13]. In particular, the QSL phase grows in stability as $V$ is increased from zero up to the point $V=K$, beyond which (for $V>K$ ) the system undergoes a first order transition to a degenerate set of classical unflippable states [13], which includes the six ordered $\langle 100\rangle$ ferromagnetic ground states described above.

In the case relevant here, when $J_{(3)} / K$ is sufficiently large, the system must undergo a transition to the unflippable $\langle 100\rangle$ ground states. Since this model and the RK one of Ref. [13] agree on the phases both when $J_{(3)} \ll K$ and when $J_{(3)} \gg K$, it is natural to expect that the intervening phase diagram coincides in the two models as well. Therefore, we expect that the QSL state is maximally stable when $J_{(3)} / K$ takes some value of $O(1)$ (for the values of the exchange constants given by our fits we find $J_{(3)} / K=\frac{J_{z \pm}^{2} J_{z z}}{4 J_{+}^{3}}=6.2$, but we caution that this is probably outside the perturbative regime). Unfortunately, there is no simple relationship between $V$ and $J_{(3)}$. However, we note that the $J_{(3)}$ exchange can also be expressed in terms of purely plaquette interactions, which might allow further analytical connection to the RK theory. We will not, however, pursue this further here.

Inclusion of the other coupling $J_{ \pm \pm}$, higher order effects, and cross terms amongst the exchange couplings does not lead to any new effects. Indeed, all the associated terms in the effective Hamiltonian assume a ferromagnetic or ring form, and can be subsumed in the above couplings. They are also higher order in $J_{\alpha} / J_{z z}, \alpha= \pm, z \pm, \pm \pm$.

\section{APPENDIX H: COMPARISON BETWEEN OUR EXCHANGE CONSTANTS AND THOSE OF THOMPSON ET AL.}

The correspondence between our effective spin-1/2 operators $\mathbf{S}_{i}$ in Eq. (1) and the full 7/2-angular-momentum operators $\mathbf{J}_{i}$ used by Thompson et al. in Ref. [10] is given by projecting the full angular momentum into the groundstate Kramer's doublet

$$
P_{1 / 2} \mathbf{J}_{i} P_{1 / 2}=\frac{\mathbf{g}_{i} \cdot \mathbf{S}_{i}}{g_{J}}
$$

where $P_{1 / 2}$ is the projection operator to the ground-state Kramer's doublet, and $g_{J}=8 / 7$ is the Landé factor.

We use $g_{x y} / g_{z}=2.4$ and $g_{z}=1.79$ for concreteness, but the results do not depend too much upon the details of this choice within the range of parameters found in the literature. With this choice, we find that the semiformal relations between our parameters and those given in Ref. [10] are

$$
\begin{aligned}
& J_{1}=0.818 \mathcal{J}_{\text {Ising }}-9.08 \mathcal{J}_{\text {iso }}-1.21 \mathcal{J}_{\text {pd }}-2.34 \mathcal{J}_{\text {DM }}, \\
& J_{2}=-0.818 \mathcal{J}_{\text {Ising }}-8.03 \mathcal{J}_{\text {iso }}-11.2 \mathcal{J}_{\text {pd }}+6.16 \mathcal{J}_{\text {DM }}, \\
& J_{3}=0.818 \mathcal{J}_{\text {Ising }}+4.88 \mathcal{J}_{\text {iso }}+12.7 \mathcal{J}_{\text {pd }}-2.34 \mathcal{J}_{\text {DM }}, \\
& J_{4}=0.818 \mathcal{J}_{\text {Ising }}-0.523 \mathcal{J}_{\text {iso }}-5.49 \mathcal{J}_{\text {pd }}+5.62 \mathcal{J}_{\text {DM. }}
\end{aligned}
$$

For $\mathcal{J}_{\text {I sing }}=6.98 \times 10^{-2} \mathrm{meV}, \mathcal{J}_{\text {iso }}=1.90 \times 10^{-2} \mathrm{meV}$, $\mathcal{J}_{\mathrm{pd}}=-2.50 \times 10^{-2} \mathrm{meV}, \quad$ and $\quad \mathcal{J}_{\mathrm{DM}}=-2.33 \times$ $10^{-2} \mathrm{meV}$ as calculated by Thompson et al. in Ref. [10], we obtain $J_{1}=-0.03 \mathrm{meV}, J_{2}=-0.07 \mathrm{meV}, J_{3}=$ $-0.11 \mathrm{meV}$, and $J_{4}=0.05 \mathrm{meV}$, or, in the formulation of Eq. (4), $\quad J_{z z}=1.9 \times 10^{-4} \mathrm{meV}, \quad J_{ \pm}=3.0 \times$ $10^{-3} \mathrm{meV}, \quad J_{ \pm \pm}=3.8 \times 10^{-2} \mathrm{meV}, \quad$ and $J_{z \pm}=$ $-6.4 \times 10^{-2} \mathrm{meV}$. These values are rather different from those found in our fits, Eqs. (3) and (5), and indeed yield spin wave spectra in strong disagreement with experiment. In principle, one resolution of the difference could be that the exchange couplings are actually strongly temperature dependent, and distinctly different in the temperature range studied in Ref. [10] and at the low temperatures studied here. Such a change in exchange parameters could conceivably occur if the transition observed at 214-240 mK had a substantial structural component. However, we do not have any a priori reason to suspect this. In any case, it would be very interesting to resolve the differences between the two exchange models.

[1] J.S. Gardner, M. J.P. Gingras, and J.E. Greedan, Magnetic Pyrochlore Oxides, Rev. Mod. Phys. 82, 53 (2010).

[2] S. T. Bramwell and M. J.P. Gingras, Spin Ice State in Frustrated Magnetic Pyrochlore Materials, Science 294, 1495 (2001).

[3] J. P. Clancy, J. P. C. Ruff, S. R. Dunsiger, Y. Zhao, H. A. Dabkowska, J. S. Gardner, Y. Qiu, J. R. D. Copley, T. Jenkins, and B.D. Gaulin, Revisiting Static and Dynamic Spin Ice Correlations in $\mathrm{Ho}_{2} \mathrm{Ti}_{2} \mathrm{O}_{7}$ with Neutron Scattering, Phys. Rev. B 79, 14408 (2009).

[4] C. Castelnovo, R. Moessner, and S. L. Sondhi, Magnetic Monopoles in Spin Ice, Nature (London) 451, 42 (2008).

[5] T. Fennell, P. P. Deen, A. R. Wildes, K. Schmalzl, D. Prabhakaran, A.T. Boothroyd, R.J. Aldus, D.F. McMorrow, and S.T. Bramwell, Magnetic Coulomb Phase in the Spin Ice $\mathrm{Ho}_{2} \mathrm{Ti}_{2} \mathrm{O}_{7}$, Science 326, 415 (2009).

[6] L.D. C. Jaubert and P. C. W. Holdsworth, Signature of Magnetic Monopole and Dirac String Dynamics in Spin Ice, Nature Phys. 5, 258 (2009).

[7] S. H. Curnoe, Structural Distortion and the Spin Liquid Sate in $\mathrm{Tb}_{2} \mathrm{Ti}_{2} \mathrm{O}_{7}$, Phys. Rev. B 78, 094418 (2008).

[8] H.R. Molavian, M.J.P. Gingras, and B. Canals, Dynamically Induced Frustration as a Route to a Quantum Spin Ice State in $\mathrm{Tb}_{2} \mathrm{Ti}_{2} \mathrm{O}_{7}$ via Virtual Crystal Field Excitations and Quantum Many-Body Effects, Phys. Rev. Lett. 98, 157204 (2007).

[9] J. D. Thompson, P. A. McClarty, and M. J. P. Gingras, Local Susceptibility of the $\mathrm{Yb}_{2} \mathrm{Ti}_{2} \mathrm{O}_{7}$ Rare-Earth 
Pyrochlore Computed from a Hamiltonian with Anisotropic Exchange, J. Phys. Condens. Matter 23, 164219 (2011).

[10] J. D. Thompson, P. A. McClarty, H. M. Rønnow, L.P. Regnault, A. Sorge, and M.J.P. Gingras, Rods of Neutron Scattering Intensity in $\mathrm{Yb}_{2} \mathrm{Ti}_{2} \mathrm{O}_{7}$ : Compelling Evidence for Significant Anisotropic Exchange in a Magnetic Pyrochlore Oxide, Phys. Rev. Lett. 106, 187202 (2011).

[11] J. A. Hodges, P. Bonville, A. Forget, M. Rams, K. Królas, and G. Dhalenne, The Crystal Field and Exchange Interactions in $\mathrm{Yb}_{2} \mathrm{Ti}_{2} \mathrm{O}_{7}$, J. Phys. Condens. Matter 13, 9301 (2001).

[12] B. Canals and C. Lacroix, Pyrochlore Antiferromagnet: A Three-Dimensional Quantum Spin Liquid, Phys. Rev. Lett. 80, 2933 (1998).

[13] M. Hermele, M. P. A. Fisher, and L. Balents, Pyrochlore Photons: The U(1) Spin Liquid in a $s=\frac{1}{2}$ ThreeDimensional Frustrated Magnet, Phys. Rev. B 69 , 064404 (2004).

[14] L. Balents, Spin Liquids in Frustrated Magnets, Nature (London) 464, 199 (2010).

[15] Y. Yasui et al., Ferromagnetic Transition of Pyrochlore Compound $\mathrm{Yb}_{2} \mathrm{Ti}_{2} \mathrm{O}_{7}$, J. Phys. Soc. Jpn. 72, 3014 (2003).

[16] J. A. Hodges et al., First-Order Transition in the Spin Dynamics of Geometrically Frustrated $\mathrm{Yb}_{2} \mathrm{Ti}_{2} \mathrm{O}_{7}$, Phys. Rev. Lett. 88, 077204 (2002).

[17] J. S. Gardner, G. Ehlers, N. Rosov, R. W. Erwin, and C. Petrovic, Spin-Spin Correlations in $\mathrm{Yb}_{2} \mathrm{Ti}_{2} \mathrm{O}_{7}: A$ Polarized Neutron Scattering Study, Phys. Rev. B 70, 180404 (2004).

[18] K. A. Ross, J. P. C. Ruff, C. P. Adams, J. S. Gardner, H. A. Dabkowska, Y. Qiu, J.R.D. Copley, and B.D. Gaulin,
Two-Dimensional Kagome Correlations and Field Induced Order in the Ferromagnetic XY Pyrochlore $\mathrm{Yb}_{2} \mathrm{Ti}_{2} \mathrm{O}_{7}$, Phys. Rev. Lett. 103, 227202 (2009).

[19] H. B. Cao, A. Gukasov, I. Mirebeau, and P. Bonville, Anisotropic Exchange in Frustrated Pyrochlore $\mathrm{Yb}_{2} \mathrm{Ti}_{2} \mathrm{O}_{7}$, J. Phys. Condens. Matter 21, 492202 (2009).

[20] S. Onoda, Effective Quantum Pseudospin-1/2 Model for Yb Pyrochlore Oxides, arXiv:1101.1230v1.

[21] J.S. Gardner, B.D. Gaulin, and D. McK. Paul, Single Crystal Growth by the Floating-Zone Method of a Geometrically Frustrated Pyrochlore Antiferromagnet, $\mathrm{Tb}_{2} \mathrm{Ti}_{2} \mathrm{O}_{7}$, J. Cryst. Growth 191, 740 (1998).

[22] H. W. J. Blöte, R. F. Wielinga, and W. J. Huiskamp, HeatCapacity Measurements on Rare-Earth Double Oxides $\mathrm{R}_{2} \mathrm{M}_{2} \mathrm{O}_{7}$, Physica (Amsterdam) 43, 549 (1969).

[23] A. Banerjee, S. V. Isakov, K. Damle, and Y. B. Kim, Unusual Liquid State of Hard-Core Bosons on the Pyrochlore Lattice, Phys. Rev. Lett. 100, 047208 (2008).

[24] D. S. Rokhsar and S. A. Kivelson, Superconductivity and the Quantum Hard-Core Dimer Gas, Phys. Rev. Lett. 61, 2376 (1988).

[25] T. Senthil, A. Vishwanath, L. Balents, S. Sachdev, and M.P.A. Fisher, Deconfined Quantum Critical Points, Science 303, 1490 (2004).

[26] D. L. Bergman, G. A. Fiete, and L. Balents, Ordering in a Frustrated Pyrochlore Antiferromagnet Proximate to a Spin Liquid, Phys. Rev. B 73, 134402 (2006).

[27] G.L. Squires, Introduction to the Theory of Thermal Neutron Scattering (Cambridge University Press, Cambridge, 1978).

[28] N. Shannon, O. Sikora, F. Pollmann, K. Penc, and P. Fulde, Quantum Ice, arXiv:1105.4196. 